\title{
Free Vibration of the Damping Beam Using Co-simulation Method Based on the MFT
}

\author{
D. Q. Wang, C. J. Wu and R. C. Yang \\ School of Mechanical Engineering, Xi'an Jiaotong University, Xi'an, Shanxi, China
}

(Received 6 October 2013; accepted 12 May 2014)

\begin{abstract}
The particle damping technique has been in development for several decades, and has been used successfully in many fields. However, it is difficult to predict its damping characteristics due to complex collisions and friction mechanisms, as well as high non-linear damping characteristics in dense particles. The focus of these current main achievements is centralized on the equivalent single degree of freedom (SDOF) system under free and forced vibration. In this paper, a brand new co-simulation approach for the continuum structure system based on the multiphase flow theory (MFT) of gas solid is developed by the COMSOL Multiphysics live link for MATLAB. A simple continuum structure system, (i.e., the cantilever particle damped beam) is made as an experiment. It is further shown that the damping capacity of a cantilever beam depends not only on the exerted location of the particle damper, but also the quantity of the filling. An experimental verification is performed, and an acceptable accordance is achieved between the theoretical results and the experimental data. It can be shown that the theoretical work in this paper is valid. The co-simulation method simplifies the complicated modelling problem, and offers the possibility to analyse the vibro-acoustic response prediction for complicated particle-damping composite structures.
\end{abstract}

\section{INTRODUCTION}

Granular particle damping, which is derived from the impact damping, is a promising technique of providing damping with granular particles placed in an enclosure attached to or embedded in the holes drilled in the vibrating structure. ${ }^{1,2}$ This emerging technology can perform well even in extreme temperatures (either low or high) and harsh chemical environments where traditional passive damping methods, such as the widely used viscous and viscoelastic dampers, are ineffective in particular applications. Particle damping technology has drawn the attention of many researchers in engineering and academic fields, and has been well researched for several decades, with a large volume of books and papers published on the subject. With the development of the particle damping technology in many fields, coherent computer simulation technology is also being developed. The simulation method is very convenient in investigating the effect of the system parameters on the characteristic of the particle damping without extensive trial-anderror testing. However, the popular simulation approaches in the published studies were more often focused on the single degree of freedom (SDOF) system or the equivalent SDOF under the free and forced vibration. It is noted that these current methods have been stretched beyond their normal capacity for the simulation of the continuum damping structure system. Consequently, it is desirable to develop a new simulation technology to predict the characteristics of particle damping. Even to this day, the simulation methods to evaluate the damping characteristic of the continuum structure systems, such as the plate and shell with the particle damper, are rare in recent research. The principal challenges are that their performances are highly nonlinear. There has also been some considerable research in the area of particle damping, and some analytical models have been developed on heuristic evaluations of particle damping.

The Discrete Element Method (DEM) simulation has been extensively developed over the years to evaluate the dissipative properties of granular materials. The DEM simulation can capture the complex interactions of the dissipation mechanisms in a particle damper. However, the DEM simulation suffers from a complicated dynamic model, and it is highly timeconsuming, which make it difficult to perform parametric analysis when the number of granules is large. It is very regrettable that the application field is only limited to the SDOF system, and therefore is not competent for the vibration analysis of the complicated continuity system with particle damper. Saeki ${ }^{3}$ used this method and investigated the damping behaviour of a horizontally vibrating system in which the gravity is not as important as in a vertically vibrating system. Mao and coworkers ${ }^{4}$ studied the characterization of particle damping in transient vibrations.

Friend et $a l .{ }^{5}$ developed a lumped mass approach, also referred to as the numerical algorithm, where the particles inside the cavity are assumed to form a lumped mass without consideration of collision and friction effects between particles. The particle damper is attached to the free end of a cantilevered aluminium beam,and the system is reduced to an equivalent SDOF system. The effects of acceleration amplitude and clearance inside the enclosure were studied, and the damping was found to be highly nonlinear, i.e., amplitude dependent. Such an approach is also applicable to investigate the damping performance of the multiple degree-of-freedom (MDOF) system to multi-body vibrating structure. ${ }^{6}$

Liu, et al. ${ }^{7}$ used an equivalent viscous damping model to represent the nonlinearity, which was extracted from experimental results. However, their studies were limited to the 